\title{
En torno a una nueva cultura
}

\author{
Luis Armando González ${ }^{1}$ \\ Recibido el 10 de marzo de 2017, aceptado el 30 de mayo de 2017 \\ "Me gustas cuando callas porque estás como ausente, \\ y me oyes desde lejos, y mi voz no te toca. \\ Parece que los ojos se te hubieran volado \\ y parece que un beso te cerrara la boca".
}

Pablo Neruda

\begin{abstract}
Resumen
La cultura predominante se caracteriza por valores (o más bien, antivalores), como el éxito fácil, el consumismo y el individualismo exacerbados, lo mismo que por unas ansias nunca satisfechas en pos de bienes materiales (dinero, principalmente), que erosionan el vínculo social porque estimulan una competencia feroz entre las personas. Es claro que el deterioro de la convivencia social en El Salvador no obedece exclusivamente a dinámicas culturales, pero éstas son parte del problema. Y, en consecuencia, son también parte de la solución. En este sentido, es necesario no sólo discutir y analizar a fondo la lógica perversa de la cultura predominante, fraguada al calor de un mercantilismo neoliberal globalizado, sino reflexionar en torno a aquellos valores que pueden ser un antídoto para unos hábitos, formas de ver la vida y prácticas que han mostrado ser perniciosas para la convivencia social y para la felicidad de las personas. Ese es el propósito de estas notas, las cuales apuntan a recuperar cuatro valores esenciales para una vida civilizada y decente: la honradez, la prudencia, el silencio y la soledad. Antes del examen y justificación de cada uno de esos valores se hace una mirada general de la cultura del Buen Vivir, en cuyo marco cobran legitimidad los valores mencionados y otros que les son complementarios.
\end{abstract}

\section{Palabras clave}

Cultura, Buen Vivir, paradigma humanista, honradez, prudencia, silencio, soledad.

1. Licenciado en Filosofía por la Universidad Centroamericana José Simeón Cañas (UCA), Maestro en Ciencias Sociales por FLACSO, México. luisinde61@gmail.com 


\begin{abstract}
The predominant culture is characterized by values (or rather, anti-values), such as easy success, consumerism and individualism exacerbated, as well as by anxieties never satisfied for material goods (money, mainly), which erode the social bond because they stimulate fierce competition between people. It is clear that the deterioration of social coexistence in $\mathrm{El}$ Salvador is not exclusively due to cultural dynamics, but these are part of the problem. And, consequently, they are also part of the solution. In this sense, it is necessary not only to discuss and analyze in depth the perverse logic of the prevailing culture, forged in the heat of a globalized neoliberal mercantilism, but to reflect on those values that can be an antidote to some habits, ways of seeing the life and practices that have shown to be harmful for social coexistence and for the happiness of the people. That is the purpose of these notes, which aim to recover four essential values for a civilized and decent life: honesty, prudence, silence and solitude. Before examining and justifying each of these values, a general view of the Buen Vivir culture is made, in which the values mentioned and others that complement them are legitimized.
\end{abstract}

\title{
Keywords
}

Culture, good living, humanist paradigm, honesty, prudence, silence, solitude.

\section{Por una cultura del Buen Vivir}

Las personas se comportan de determinada manera, toman determinadas decisiones, a partir de sus creencias, valores, formas de ver la vida, visiones ideológicas, tradiciones y conocimientos; todo ello se engloba bajo la noción de cultura. La cultura de una sociedad no se fragua desde una sola vertiente, sino desde muchas: sistema educativo, iglesias, instituciones, élites, empresas... pero, sobre todo, desde las prácticas concretas que las personas realizan en su cotidianidad, en sus interacciones sociales, en su participación comunitaria, en su vida familiar. Por eso la raíz de la palabra cultura es "cultivo"; y se cultiva desde la práctica, no desde la teoría. En el caso de los valores (y su contracara: los antivalores), tan cruciales para la toma de decisiones individuales y colectivas, es su cultivo práctico en el que cuenta, pues su función es orientar y justificar las opciones y comportamientos cotidianos de las personas. No hay modo de dar vida a nuevos valores - de superar antivalores dañinos para la dignidad humanasi estos no surgen de experiencias prácticas que los sostengan y los hagan viables y efectivos también en la práctica.

El Buen Vivir, como paradigma humanista que pone a la persona humana, en su integralidad individual y social, en el centro del quehacer estatal, supone y 
exige el cultivo de nuevas creencias, costumbres, hábitos, formas de ver la vida, actitudes y valores que orienten y den la pauta a comportamientos y dinámicas de relación social distintas a las predominantes. La cultura predominante individualista, competitiva, egoísta, dependiente, sumisa y poco responsableno dignifica a las personas, coarta su creatividad, su compromiso, la conciencia de sus derechos, la preocupación por los demás, la solidaridad, la cooperación y la fraternidad.

Es esa cultura predominante la que ha comenzado a romperse en el país, desde que el Buen Vivir comenzó a cultivarse en 2014. Estamos viendo la fragua, incipiente ciertamente, de una cultura del servicio hacia la sociedad -especialmente hacia los más débiles- que emana del gobierno, pero que está llamada a permear a todo el aparato estatal. Se ha hablado hasta la saciedad del "servicio público", pero es hasta ahora que en realidad está cobrando vida la idea de que el funcionario público es un servidor de la sociedad, que su trabajo es responder a los problemas de la gente, buscarles la mejor solución. El Presidente Salvador Sánchez Cerén es el ejemplo mejor de esta nueva cultura del servicio público que, si arraiga como debiera, hará del Estado una esfera de poder al servicio de la sociedad, y no a la inversa como suele ser en la cultura tradicional.

Asimismo, se está gestando una cultura de la participación distinta la vigente, caracterizada fuertemente por ser: a) una participación restringida a lo electoral; b) una participación esporádica, es decir, no sostenida en el tiempo por una institucionalidad permanente; y c) una participación de demandas, esto es, de pedir al Estado bienes y servicios, sin compromisos ciudadanos como contrapartida.

La cultura de participación predominante tiene un impacto nocivo sobre la responsabilidad ciudadana en distintos ámbitos de la vida familiar, comunitaria y social. En esa cultura, siempre son otros -siempre son los funcionarios y el mismo Estado- los responsables de las diferentes problemáticas que aquejan a la familia, la comunidad y la sociedad. Y si es así, son ellos los que deben resolver esos problemas; los ciudadanos y ciudadanas, por su parte, nada más deben esperar a que ello suceda y, si no sucede, reclamar, denunciar y quejarse.

Definitivamente, esa visión desvincula a los ciudadanos y ciudadanas de aquellas responsabilidades que sí son de ellos y ellas; elimina la corresponsabilidad -es decir, la articulación entre la sociedad y el Estado para atender determinadas problemáticas-; y hace de todos los problemas sociales, culturales, políticos y económicos un asunto del Estado, especialmente del Ejecutivo. La nueva cultura de la participación, propia del Buen Vivir, cultiva la responsabilidad ciudadana, la corresponsabilidad ciudadanos-Estado y la responsabilidad propia del Estado en aquellos ámbitos que le corresponde a cada cual. 
En este marco, la responsabilidad de los ciudadanos es un valor cultural que, aunque es incipiente, será decisivo para darle un nuevo rumbo al país. Esa responsabilidad tiene que ver, sin duda, con la conciencia de los propios derechos y la decisión de hacerlos valer, pero también con la conciencia de los deberes ineludibles que hay que cumplir para vivir en una sociedad más armónica y acogedora. Sin este último requisito -que se expresa en la obligación de todo salvadoreño y salvadoreña de no hacer daño a los demás, de cuidar su entorno de vida natural y social, de respetar las leyes, de ser justos, tolerantes, de atender a los débiles, de no violentar a los demás- no será posible una mejor sociedad.

Lo que cultiva la cultura del Buen Vivir es una nueva forma de vernos y de actuar en la sociedad, una nueva forma de relacionarnos con la naturaleza y con nuestros semejantes. Cuando nos apropiamos de los valores que promueve el Buen Vivir, inmediatamente asumimos que lo que hagamos o dejemos de hacer crea un daño o un bien a los demás, y a nosotros mismos y nuestra familia. Si violamos los derechos de los demás, si violentamos a otros, si no respetamos las leyes, si somos intolerantes, si discriminamos, si no obramos rectamente... si hacemos todo eso (y más) estamos, personalmente, trabajando por el mal vivir en la sociedad, un mal vivir del cual, además de víctimas, somos responsables directos o indirectos.

¿Superaremos en algún momento esa cultura del mal vivir? Depende de nosotros; depende del Estado; depende de los empresarios; depende de los medios de comunicación; depende de las universidades, las iglesias, los gremios, los sindicatos y las asociaciones profesionales. En fin depende, de muchas voluntades. Depende de que cada quien haga, responsablemente, lo que le corresponde. Ahora bien, esa responsabilidad, que en sí misma es un valor a cultivar, debe nutrirse de un entramado de valores entre los cuales destacan la honradez, la prudencia, el silencio y la soledad.

\section{La honradez}

Una expresión que se escuchaba con frecuencia en épocas pasadas era "pobre, pero honrado". Bien se podía decir de forma individual - "Yo soy una persona pobre, pero honrada"- o de forma grupal - "Somos pobres, pero honrados". Algo profundo se apuntaba con la frase en cuestión: la honradez era algo sumamente importante, tanto que se remarcaba su presencia en esa condición límite que es la pobreza. Se trataba en ese entonces de la vigencia de un valor -el de la honradez- que servía de norte moral en la vida de las personas. Era parte de un conjunto de valores y preceptos que nutrieron el clima moral de la sociedad salvadoreña a lo largo del siglo XX, hasta esa década de cambio cultural y económico que fue la de los noventa de ese mismo siglo. 
La honradez convivía extraordinariamente bien con el honor, la cortesía, la hospitalidad y la cordialidad, con las cuales formaba un amasijo de prácticas, usos, costumbres y estilos de comportamiento que daban solidez al tejido social en barrios, colonias, pueblos y ciudades. No es que en esas décadas no hubiera personas poco honradas o para nada honradas (o sin honor, descorteses, no hospitalarias o poco cordiales); por supuesto que sí. Pero en el clima cultural prevaleciente, la honradez (y el conjunto de valores del que ella formaba parte) era reconocida como norma de conducta y criterio de valoración de comportamientos y actitudes.

De tal suerte que alguien que hiciera algo deshonroso (o algo falto de honor, etc.) era consciente, ante sí mismo y ante los demás, de su falla. Es decir, su conciencia moral y el juicio moral de los otros asumían que era reprochable haber violado la exigencia socialmente compartida de la honradez y de ello se seguían las actitudes y comportamientos correspondientes: de vergüenza por parte de la persona que había cometido el acto bochornoso y de regaño, rechazo e incluso ostracismo por parte de familiares, amigos y comunidad.

En el marco de un cambio económico y cultural que se comenzó a fraguar en los años ochenta, pero con más contundencia en los años noventa, la honradez (y los valores que le estaban asociados) comenzó a desfallecer como valor moral importante (como criterio normativo y valorativo de la conducta de la gente). Desfalleció hasta prácticamente desaparecer del horizonte moral de la sociedad salvadoreña.

Otro clima moral comenzó a ganar predominancia (hasta imponerse casi absolutamente), a la par de la gestación de una mercantilización económica que, al ponerle precio a todo y medir todo a partir de criterios de pérdidas o ganancias económicas, hizo de la honradez algo poco redituable en términos económicos. Ese nuevo clima cultural y el mercantilismo, que se impuso desaforadamente a partir de los años noventa, soterraron (anularon, desacreditaron, ridiculizaron) el valor de la honradez. En este marco, la satisfacción moral que se deriva de la honradez dejó de ser importante.

El éxito fácil (económico, principalmente), la ostentación (carros, casas, joyas, viajes), la acumulación de bienes, el ascenso socio-económico, el consumismo exacerbado, las expectativas infladas acerca de lo que se merece en términos económicos (pero no sólo económicos), la insatisfacción permanente con lo que se ha logrado en bienes materiales y la búsqueda permanente de más cosas (más lujosas, más caras, más ostentosas), el desprecio hacia los débiles y hacia los poco ambiciosos (considerados "fracasados"), la adulación hacia los "triunfadores", sobre todo si su triunfo había sido fácil, la contraposición entre los "vivos" y los tontos, esos y otros valores de igual calado se fueron imponiendo lenta pero inexorablemente en amplios sectores de la sociedad, especialmente en la clase media. 
En una palabra, la vida de los ricos más ricos se convirtió en el modelo a seguir, en aspiraciones, búsqueda de dinero, consumo y ostentación. Ser como los ricos (pertenecer a la clase de los nuevos ricos) se impuso como el supremo ideal una clase media huérfana de otros referentes, hastiada de renuncias y compromisos, y ansiosa, ahora sí, de ascender hacia el sitial de privilegios del que históricamente había sido marginada. Los ricos parecían dispuestos de darle el dinero que hiciera falta (con préstamos y tarjetas de crédito) y las oportunidades para disfrutar de la felicidad que sólo el dinero puede dar. Alguien dijo, por aquellos años noventa: "el dinero no es toda la felicidad, pero casi", con lo cual expresaba el espíritu de los nuevos tiempos para la clase media.

Ciertamente, a nuestra clase media, desde su constitución firme (o bastante firme) a mediados de los años cincuenta del siglo XX, nunca le fueron ajenos algunos de esos valores o valores parecidos. El afán de progresar, la búsqueda del ascenso social, el poseer bienes y recursos generados en el marco de la urbanización y la industrialización..., pero esos valores no anularon valores importantes como la honradez (o la solidaridad, el compromiso con los sectores populares, la hospitalidad, el compartir, etc.), pues antes de la década de los noventa la clase media salvadoreña no tenía como aspiración exclusiva suya vivir en la riqueza a la manera de los ricos del país ni tampoco el mercantilismo la había atrapado en sus redes como lo hizo desde que finalizó la guerra civil.

La clase media abrazó el nuevo clima cultural sin reserva alguna. Y se plegó al mercantilismo sin rechistar e incluso con satisfacción. Renunció a derechos -como la educación pública, por ejemplo- que la habían beneficiado a ella principalmente, pero también a los sectores populares. Aceptó la lógica de que la educación privada es mejor (según el lema que dice "cuanto más caro es algo es de mayor calidad"), porque vio en ello un signo de distinción y ascenso socio-económico.

De la misma manera se hizo cargo de la mercantilización de la salud, las pensiones y las telecomunicaciones. Asumió sin rechistar que no había nada más importante y mejor en la vida que ser una persona rica y que para lograr tal propósito cualquier medio era bueno. Lo importante era triunfar, tener éxito. Sobresalir. Cualquier cosa que se interpusiera en el camino debía ser desechada como una jugarreta de quienes estaban en el mismo empeño aunque dijeran lo contrario.

Fue así como la honradez fue expulsada de la vida cultural y social. Y es que para quienes estaban atrapados en las redes del éxito fácil, la acumulación de dinero y el mercantilismo, la honradez o era abanderada por los tramposos (que aspiraban a lo mismo) o por los “perdedores", los “dinosáuricos”, los que no entendían que quien no tiene dinero no vale nada. 
Las consecuencias de haber desterrado la honradez de la vida cultural y social (lo mismo que haber desterrado los valores que le son próximos) han sido graves consecuencias para la convivencia social. Ninguna sociedad puede apostarle a un proyecto de convivencia decente (igualitario, de bienestar compartido, de solidaridad) si amplios sectores suyos están dispuestos a lo que sea con tal de convertirse en ricos de la noche a la mañana. Un valor como la honradez es un correctivo a ese tipo de conductas, un correctivo individual pero también un correctivo social.

Por eso, en esta reflexión se hace una defensa de la honradez. Pero junto a ella se defienden otros valores que la cultura neoliberal ha anulado y que es necesario revitalizar, tal como han hecho otras naciones en el mundo.

A propósito de lo anterior, en un reciente documental de Michael Moore, titulado “¿Qué invadimos ahora?”, el cineasta viaja por Europa en busca de bienes de conquista para llevar a su país. Se hace referencia aquí a tres países, como ilustración: en Italia, en una empresa de ropa los dueños le dicen que para ellos sus trabajadores deben gozar de una buena vida, y que eso no pone en riesgo sus ganancias; en Portugal, los policías le dicen que lo más importante en su país es la dignidad de las personas; y en Finlandia, donde los niños reciben pocas horas de clase y gozan de tiempo para jugar, los maestros le hacen ver que lo más importante es que los niños no dejen de serlo. Dignidad, felicidad, amistad, tolerancia, fraternidad y respeto a quienes nos rodean versus consumismo, afán de triunfar, sobresalir opacando a otros, poseer cosas (dinero, carros, casas) y ostentar. Definitivamente, los finlandeses, los italianos o los portugueses viven mejor (son más felices, más dignos) que los estadounidenses. Esa es la conclusión de Michael Moore. Definitivamente, para nosotros, apropiarnos del "estilo americano" fue una pésima elección.

\section{La prudencia}

Acabamos de hablar de la honradez. Ahora le toca el turno a la prudencia, una de esas virtudes esenciales que hoy por hoy, al igual que la honradez y sus valores asociados, está de capa caída. Soterrada por la imprudencia -manifestada de mil y una formas - la prudencia urge de ser revitalizada, pues sin ella, como autocorrectivo individual y colectivo, el sentido de los límites y las precauciones ante los propios excesos se pierden de manera inexorable. $Y$ es que precisamente a lo que apunta la prudencia es la autocontención, al autocontrol, a evitar los excesos en acciones y palabras, a no traspasar ciertos límites, a evitar los extremos en lo que hace o en lo que se dice.

Para Cicerón, la prudencia es, junto con la justicia, la fortaleza y la templanza, uno de los cuatro principios de la honestidad. Y sobre ello apunta lo siguiente: 


\begin{abstract}
"Mas todo lo que es honesto ha de proceder de alguna de estas cuatro partes. Porque, o consiste en la investigación y conocimiento de la verdad, o en la conservación de la sociedad humana, en dar a cada uno lo que es suyo, y en la fidelidad de los contratos, o en la grandeza y firmeza de un ánimo excelso e invencible, o en el orden y medida de todo cuanto se dice y hace, en que se comprende la moderación y la templanza.
\end{abstract}

Estas cuatro partes, aunque están unidas y enlazadas entre sí con una mutua dependencia, con todo, cada una de ellas produce ciertas clases de obligaciones particulares. Por ejemplo, de la primera, en que colocamos la prudencia y la sabiduría, nace la indagación y el descubrimiento de la verdad; y este es el oficio propio de esta virtud. Porque el hombre que con más claridad percibe la pura e ingenua verdad de cada objeto, el que penetra y explica con más agudeza y prontitud las razones, es el que se reputa por más sabio y prudente. Por lo cual el objeto de esta virtud y la materia, digámoslo así, que ha de tratar y en que ha de ejercitarse, es la verdad".

Prudencia no es igual a cobardía, pues quien es prudente sabe ser valiente cuando las circunstancias lo ameritan. Por tanto, lo opuesto a la prudencia no es la valentía, sino la imprudencia, bajo cuyo apartado se engloban todos aquellos hábitos y prácticas fuera de control, excesivos y sin límites. Se trata hábitos y acciones imprudentes un sentido ético-moral: dañan moralmente a otros, alteran la vida los demás, tienen implicaciones negativas en la convivencia entre las personas.

Decíamos que antes que se puede ser imprudente de palabra y de obra. Los ejemplos de uno y otro tipo abundan. En el primer caso, una persona es imprudente de palabra cuando habla en lugar de callar, cuando dice más de lo que debe decir, cuando habla para herir a otros o cuando dice o escribe cosas carentes de sentido y fuera de contexto. En nuestro país, la imprudencia verbal es un mal endémico. Las “redes sociales” están ahí para corroborarlo. También están los programas de entrevistas en la televisión y las columnas de opinión en la prensa escrita. Muchas veces, quienes son imprudentes en el uso de las palabras lo hacen sin la intensión de causar daño a terceros - a esas personas se aplica aquello de que "sólo hablan por hablar"-. Sin embargo, en incontables ocasiones, la imprudencia verbal sí tiene como objetivo causar daño: en los años setenta y durante casi todos los años ochenta la imprudencia verbal (por ejemplo, de los medios de comunicación en la muerte de los jesuitas de la UCA o de los llamados “orejas" que delataban a los opositores de los gobiernos militares) se tradujo en la muerte de personas inocentes. En el presente, una buena parte de las opiniones imprudentes que se escuchan o leen tienen por finalidad causar daño al gobierno, al FMLN o a dirigentes de este partido. 
En cuanto a acciones imprudentes, las hay en abundancia. A los conductores de vehículos particulares, microbuses y autobuses se suman ahora los conductores de motocicletas, a quienes casi en bloque les ajeno el sentido de los límites y del autocontrol. Su imprudencia, en este caso, pone en riesgo la integridad física de quienes caminan, ciertamente con dificultades, por las calles y avenidas de las ciudades del país, principalmente en San Salvador. Hay, por supuesto, otras acciones que se pueden ser leídas desde el término que nos ocupa. Por ejemplo, el evento de jóvenes de ARENA auspiciado por la embajada de EEUU en días recientes, fue un acto imprudente por parte de la embajadora de ese país en El Salvador.

Naturalmente que esa acción debe ser evaluada desde otros criterios, políticos y jurídicos. Desde este punto de vista, se trató de un acto partidario ilegítimo e injustificable, en cuanto que un representante diplomático acreditado ante el Estado salvadoreño no debe (ni puede) identificarse abiertamente con un partido ni mucho menos sumarse a su campaña política. Y eso fue lo que hizo la embajadora de EEUU, Jean Manes, al patrocinar un evento con y para la juventud de ARENA. Es impensable que la embajada de El Salvador en EEUU (o cualquier embajada de otro país) realice un acto político partidario en su sede diplomática en favor de un partido en contienda en EEUU. La embajadora cometió un grave error diplomático, y su acción está a la espera de un análisis serio y no servil por parte de los expertos en derecho internacional.

Pero más allá de eso, la embajadora de EEUU fue imprudente al auspiciar a un grupo de jóvenes vinculados con un partido político, y cuya finalidad -como dijo el líder del movimiento- es fortalecer a ARENA. La embajadora tuvo que haberse autocontenido, tuvo que haberse puesto límites a sí misma, pues hay cosas que ella no puede hacer, por más que las ganas la desborden. Son imprudentes también quienes la defienden, sabiendo que lo que ella hizo es indefendible. Apelar a los derechos de los jóvenes a expresarse políticamente es una defensa inadecuada (imprudente, porque se dice algo fuera de contexto y ajeno al tema en discusión) pues un partido como ARENA (o cualquier partido) debe buscar los medios para canalizar las ansias de su juventud. Además, en ARENA se trata de un partido con dinero suficiente para generar esos espacios sin los auspicios de la embajada de EEUU.

Así que la imprudencia diplomática salió a relucir de manera abierta. Salió a relucir la imprudencia de quienes opinan sin argumentos de peso, sin atreverse a aceptar que hay cosas intolerables.

Cuánta falta nos hace la prudencia. Cuánta falta nos hacen esos valores que le son afines: la mesura, el silencio, el sentido del equilibrio y del tiempo, la templanza y la claridad mental (que sólo da un buen uso de la razón). Decir lo primero que se viene a la mente es un atentado contra la prudencia verbal. 0 , lo que es lo mismo, no pensar bien lo que se va a decir. Actuar sin medir 
las consecuencias de lo que se hace es un atentado contra la prudencia en las acciones. Como quiera que sea, ahí donde predomina la imprudencia (verbal o en las acciones) la convivencia con nuestros semejantes es sumamente complicada. Nuestros mayores lo sabían y por eso acuñaron expresiones de enorme sabiduría, como estas:

\title{
"En boca cerrada no entran moscas" \\ "Es mejor callar que hablar" \\ "Calladito se ve más bonito" \\ "Machete estate en tu vaina"
}

\begin{abstract}
Ahora bien, ser imprudente no cuesta nada. Ser prudente sí que es difícil; por eso es un deber ser. La imprudencia acecha por doquier, y hay que tener una dosis suficiente de razón y de equilibrio para no ser presa fácil de sus argucias. Nadie está exento de caer en sus garras en cualquier momento de su vida. Todos hemos sido imprudentes en alguna ocasión y seguramente lo volveremos a ser. Nadie puede presumir, pues, de haber sido siempre y en todo lugar un ejemplo sin par de prudencia. Pero eso no quiere decir que nos crucemos de brazos y cultivemos, sin control alguno -imprudentemente--, ese pernicioso hábito. Al contrario, la facilidad con la que se expande (y los daños que causa) en estos tiempos de chismorreo mediático hace más necesaria que nunca la tarea de cultivar la prudencia como hábito vital para una convivencia civilizada. La incrustación cultural de la imprudencia (en las acciones y las palabras) da la medida de lo difícil que es la tarea de revitalizar culturalmente la prudencia y el conjunto de valores que le son afines.
\end{abstract}

De nuevo, una cita de Cicerón:

"es obligación del ánimo constante y fuerte no perturbarse en los casos adversos ni caer de su estado, digámoslo así, por alucinarse; sino estar siempre sobre sí, y no apartarse de la razón.

Mas aunque éstas son propiedades de ánimos grandes, es también propio de mucho entendimiento el prevenir con el pensamiento lo venidero, y tener formado juicio de lo que por una y otra parte puede acontecer, y lo que se ha de hacer en cualquier acontecimiento; de forma que nada nos sorprenda... Lo cual cabe únicamente en un ánimo grande y sublime que sólo se fía y se funda en la razón y la prudencia".

\section{El silencio y la soledad}

Es poco (o casi nada) lo que se dice sobre el valor del silencio y de la soledad. A propósito del silencio, quizás porque una larga tradición autoritaria se las arregló para imponerlo como mecanismo de sometimiento y miedo. Pero no es de ese silencio “impuesto" del que aquí se trata, sino del silencio como 
una opción y una alternativa al ruido imperante y a la obligación socialmente condicionada de no permanecer en silencio, sino a sumar la propia voz al ruido predominante. Es decir, en los tiempos que corren lo obligado no es silencio que está mal visto- sino su contrario: el ruido, el bullicio. De ahí la importancia de reivindicarlo como un valor imprescindible para una vida buena, como ya lo sabían los moralistas romanos en la antigüedad clásica, los Padres de la Iglesia y los pensadores orientales.

Hermano gemelo de la prudencia, el silencio es más radical. Aquélla invita a la mesura en el decir y en el obrar, este a suspender el decir, ya sea de forma temporal o ya sea de forma definitiva, como hicieron en el pasado monjes, santos y ermitaños en la tradición cristiana. En una sociedad del bullicio como esta en la que nos toca vivir, quizás sea imposible el silencio absoluto. No lo es, sin embargo, el silencio parcial, el silencio temporal. Justamente, la sociedad del bullicio nace necesaria y urgente la puesta en vigencia una práctica del silencio que poco a poco se vaya convirtiendo en un hábito individual y colectivo que incida - junto con valores como la prudencia, la honradez, la sensatez, la tolerancia y la moderación- en las formas de comportamiento vigentes, y ayude a su transformación.

¿Silencio para qué? Para muchas cosas, francamente positivas y sanas. En primer lugar, para conversar con uno mismo, como quería Antonio Machado, quien además esperaba también hablar algún día con Dios. Así lo dice el poeta en "Retrato":

\section{"Converso con el hombre que siempre va conmigo - quien habla solo espera hablar a Dios un día-; mi soliloquio es plática con ese buen amigo que me enseñó el secreto de la filantropía".}

Qué importante y vital es conversar con uno mismo, tomarse el tiempo para hacerlo. Cuánta gente no lo hace, atrapada no sólo en la vorágine de los tiempos y ritmos impuestos por el reloj, sino dominada por las exigencias de estar, permanentemente, diciendo algo, de estar siempre volcada hacia el exterior de sí misma. Conversar con uno mismo es volcarse hacia la propia vida interior, para explorar asuntos que trascienden la inmediatez que nos devora -para abrirnos al misterio de Dios, como quiere Machado- o para pensar mejor nuestras acciones y decisiones.

Esa pausa del pensamiento reflexivo es algo que brilla por su ausencia en este país del activismo febril y del decir fácil. Para pensar reflexivamente, para conversar con nosotros mismos acerca de la realidad del más allá y del más acá, es necesario, es imprescindible, el silencio. Sin él, es imposible pensar. Y si no se piensa bien lo que se va a decir o lo que se va a hacer, lo más seguro es que se digan sinsentidos y absurdos y que se realicen acciones precipitadas y fuera de control. 
En segundo lugar, silencio para resguardarse, para protegerse, de las inclemencias de un entorno hostil y pernicioso para la salud mental y corporal de las personas. En la tradición budista esto es evidente. También lo es en las tradiciones hinduistas y confucianas que reconocieron el valor esencial del silencio para el cuido de la vida mental y corporal. Las enseñanzas budistas lo apuntan con claridad:

\begin{abstract}
"Tu silencio interno te vuelve sereno. Haz regularmente un ayuno de la palabra para volver a educar al ego. Practica el arte de no hablar.

Progresivamente desarrollarás el arte de hablar sin hablar y tu verdadera naturaleza interna reemplazará tu personalidad artificial dejando brotar la luz de tu corazón y el poder de la sabiduría el 'noble silencio'. Gracias a esta fuerza atraerás hacia ti todo lo que necesitas para realizarte y liberarte. Así pues, quédate en silencio".
\end{abstract}

Quedarse en silencio: un imperativo que, de convertirse en criterio de autocontrol personal, redundaría una mejor condición de salud mental y corporal. Pues, precisamente, el entorno ruidoso que nos golpea cotidianamente no sólo llega a nuestra mente, sino también a nuestro cuerpo. Nuestros sentidos y nuestra sensibilidad se han acostumbrado tanto a la música estridente, los gritos, la publicidad y las arengas de los activistas religiosos (que en conjunto generan un bullicio fuera de control y difícil de procesar) que no nos damos cuenta del deterioro que eso supone para nuestra calidad de vida. Se ha hecho tan normal la invasión y laceraciones al propio espacio mental y corporal que no nos damos cuenta de lo anómalo de la situación. No nos damos cuenta del impacto de ello en nuestra felicidad personal y familiar. Sólo nos daremos cuenta de la gravedad de tales dinámicas en la medida en que comencemos a valorar el silencio. Y, en la que medida que lo vayamos poniendo en práctica, no daremos cuenta de su importancia como resguardo ante las inclemencias de un entorno ruidoso y avasallador de nuestra integridad personal.

El silencio como resguardo nos permite defender y ejercer un doble derecho: a) el derecho a guardar silencio cuando no queremos hablar y b) el derecho a no escuchar aquello que no queremos escuchar. Lo primero va a contracorriente de mala costumbre predominante de (casi) forzar a que la gente siempre diga algo, aunque esté fuera de lugar, sea reiterativo o carezca de sentido. Contra toda razón, el silencio se ve mal, no se tolera. El ruido se estimula y premia. Se tiene que invertir este estado de cosas, dando su debido lugar al silencio no sólo como un hábito loable, sino como un derecho de quienes simplemente prefieren callar en lugar de hablar. Lo segundo va a contracorriente de la mala costumbre de obligar a otros que escuchen lo que no quieren escuchar. Desde quienes hablan por teléfono celular delante de quien sea, pasando por quienes lanzan consignas religiosas en las plazas y parques, hasta los conductores de motos y carros que pitan descontroladamente en calles y avenidas, la imposición de 
ruidos hirientes para la sensibilidad y la mente es generalizada y preocupante. En relación con lo anterior, en tercer lugar, el silencio es un importante recurso para no interferir abusivamente en el espacio mental, pero también físico de los otros. O sea, el silencio nos protege de los demás, pero también los protege a ellos de nosotros. Se trata, en ese sentido, de un mecanismo de autocontrol, ante la propia vocación a ser ruidosos y a inmiscuirnos en la vida interior de los demás, violentando muchas veces el derecho al silencio que ellos nos hacen. Hijos e hijas de una cultura del bullicio y del ruido, hijos e hijas de una cultura que castiga el silencio y alienta el no quedarse callados, nosotros también debemos "practicar al arte de no hablar" para no interferir en el silencio de otros o para ayudarles a cultivarlo.

Tenemos que respetar el derecho de los demás no solo a quedarse callados cuando no quieren hablar - sin cuestionar ni inmiscuirnos en las razones de ello- sino también a no ser invadidos, en su intimidad mental y corporal, por nuestras arremetidas ruidosas mediante las palabras que salen de nuestra boca o mediante ruidos que salen de instrumentos bajo nuestro poder (carros, motos, video caseteras, televisión, radio o aparatos de música). Violar el derecho de otros al silencio y al resguardo de su intimidad debería ser algo intolerable para uno mismo, así como también debería serlo que otros atentaran contra ese derecho en nosotros.

Terminamos con una breve reflexión sobre la soledad, que por cierto está íntimamente ligada al silencio. Al igual que éste, también la soledad está de capa caída en estos tiempos ruidosos y mercantilistas. Es difícil conversar con uno mismo en silencio -o leer un libro o meditar o reflexionar sobre asuntos que van más allá de lo inmediato- sin una dosis de soledad, pues el ruido y la interferencia de los demás no son buenos acompañantes de hábitos como los mencionados. Pero no sólo se trata de una soledad que es necesaria para el cultivo de actividades intelectuales o meditativas, sino que también lo es para el cuido de la integridad corporal, lo cual por el cultivo de un espacio (o de un ámbito) al cual sólo debe tener acceso exclusivo la propia persona, y quizás sólo extraordinariamente aquellos a los que ella autorice.

A primera vista, es curioso que, en un mundo en el que se proclama como la esencia de todo el individualismo y la privatización, la soledad tenga tan poca valía. Hay una razón para ello: el individualismo privatizador es ruidoso, violenta la integridad física y mental de las personas, invade con sus productos tecnológicos la intimidad de la gente.

La soledad es imposible en un contexto cultural y económico en el cual las personas están atrapadas en las redes de comunicación y de consumo casi las 24 horas del día, los 7 días de la semana. Lo que menos desean los estrategas de la economía y la cultura neoliberales es que las personas busquen refugio en 
la soledad (o en el silencio). Eso significaría una parálisis para el consumismo, que pasa por una renuncia al autodominio, al auto cuido y a la propia integridad mental y corporal, y una obsesión por las relaciones incesantes con el mercado y sus productos.

El individualismo privatizador es masificador; uniformiza los gustos y los hábitos. Avasalla al individuo, no lo reivindica. Anula los ámbitos de la vida privada en los que se juega la intimidad y la dignidad de las personas. Los mercantiliza. Los publicita. Como nunca, en estos tiempos neoliberales, la vida privada de las personas se ha desvanecido, ante los embates de una cultura que fomenta esa renuncia a lo que debería ser exclusivo de cada persona en particular.

Es por lo anterior que la soledad y el silencio se hacen necesarios. No como valores absolutos. No de forma dogmática. Hay que tomarlos como recursos terapéuticos en estos tiempos tan urgidos de contrapesos a sus prácticas, hábitos y costumbres que avasallan a la gente y que ponen en vilo su salud mental y corporal.

\section{Reflexión final}

Las dinámicas culturales inciden fuertemente en la convivencia social, erosionándola o fortaleciendo el nexo social. De ahí la importancia de reflexionar en torno a la cultura predominante en una sociedad como la salvadoreña, caracterizada por un grave deterioro en los valores, los hábitos y las prácticas individuales y colectivas. Es decir, esta reflexión es urgente cuando en la sociedad tienen vigencia dinámicas culturales que socavan las posibilidades de unos valores, hábitos y prácticas respetuosos de la dignidad de las personas y del entorno socio-natural, y propiciadores del florecimiento de estilos de vida anclados en el apego a la comunidad y en la memoria histórica. Nuevos valores, nuevas creencias, nuevos hábitos y nuevas prácticas se hacen necesarios en un contexto como el de El Salvador de la segunda década del siglo XXI, en el cual la cultura predominante, consumista, mercantilista, individualista y neoliberal, está lejos de ser el cemento que contribuya a su viabilidad como un proyecto de convivencia social solidario, justo e inclusivo, el cual el eje legitimador del quehacer estatal y empresarial sea la dignidad de las personas y no la acumulación y/o la ostentación de bienes y riqueza.

\section{Bibliografía}

Cicerón, Los oficios. México, W.M. Jackson, 1973.

Díaz, C., Manual de historia de la religiones. Bilbao, Desclée De Brouwer, 1999.

Savater, F., Diccionario filosófico. Barcelona, Planeta, 1995.

Singer, P. (Ed.), Compendio de ética. Madrid, Alianza, 2000.

Storing, H. J., Historia universal de la filosofía. Madrid, Tecnos, 2000. 